\title{
AN APPROXIMATIVE SCHEME OF FINDING ALMOST HOMOCLINIC SOLUTIONS FOR A CLASS OF NEWTONIAN SYSTEMS
}

\author{
JOANNA JANCZEWSKA
}

\begin{abstract}
In this work the problem of the existence of almost homoclinic solutions for a Newtonian system $\ddot{q}+V_{q}(t, q)=f(t)$, where $t \in \mathbb{R}$ and $q \in \mathbb{R}^{n}$, is considered. It is assumed that a potential $V: \mathbb{R} \times \mathbb{R}^{n} \rightarrow \mathbb{R}$ is $C^{1}$-smooth with respect to all variables and $T$-periodic in a time variable $t$. Moreover, $f: \mathbb{R} \rightarrow \mathbb{R}^{n}$ is a continuous bounded square integrable function and $f \neq 0$. This system may not have a trivial solution. However, we show that under some additional conditions there exists a solution emanating from 0 and terminating to 0 . We are to call such a solution almost homoclinic to 0 .
\end{abstract}

\section{Introduction}

The goal of this paper is to establish the existence of an almost homoclinic solution for a class of Newtonian systems of the form:

$$
\ddot{q}+V_{q}(t, q)=f(t)
$$

where $t \in \mathbb{R}, q \in \mathbb{R}^{n}$.

2000 Mathematics Subject Classification. 37J45, 58E05, 34C37, 70H05.

Key words and phrases. Almost homoclinic solution, periodic orbit, action functional, Newtonian system.

The research is supported by the State Committee for Scientific Research, Poland, grant no. 1/P03A/042/29. 
Definition 1.1. We will say that a solution $q: \mathbb{R} \rightarrow \mathbb{R}^{n}$ of the Newtonian system (1.1) is almost homoclinic to 0 , if $q(t) \rightarrow 0$, as $t \rightarrow \pm \infty$.

Note that $q_{0} \equiv 0$ may not satisfy (1.1).

We assume that $V: \mathbb{R} \times \mathbb{R}^{n} \rightarrow \mathbb{R}$ and $f: \mathbb{R} \rightarrow \mathbb{R}^{n}$ satisfy the following conditions:

$\left(\mathrm{C}_{1}\right) V$ is $C^{1}$-smooth with respect to all variables and $T$-periodic with respect to $t, T>0$,

$\left(\mathrm{C}_{2}\right) f \neq 0$ is bounded, continuous and square integrable.

Our approach to (1.1) involves the use of a variational method of an approximative nature. To formulate a theorem, let $E:=W^{1,2}\left(\mathbb{R}, \mathbb{R}^{n}\right)$ be a Hilbert space under the standard norm

$$
\|q\|_{E}:=\left(\int_{-\infty}^{\infty}\left(|q(t)|^{2}+|\dot{q}(t)|^{2}\right) d t\right)^{1 / 2} .
$$

For every $k \in \mathbb{N}$, let $f_{k}: \mathbb{R} \rightarrow \mathbb{R}^{n}$ be a $2 k T$-periodic extension of $f$ restricted to the interval $[-k T, k T)$ over $\mathbb{R}$. Let us remark that $f_{k}$ may not be continuous at points: $k T \pm 2 k T j, j \in \mathbb{Z}$.

We consider a family of Newtonian systems

$$
\ddot{q}+V_{q}(t, q)=f_{k}(t),
$$

where $t \in \mathbb{R}, q \in \mathbb{R}^{n}$. Let $E_{k}:=W_{2 k T}^{1,2}\left(\mathbb{R}, \mathbb{R}^{n}\right)$ be a Hilbert space of $2 k T$-periodic functions with the usual norm

$$
\|q\|_{E_{k}}:=\left(\int_{-k T}^{k T}\left(|q(t)|^{2}+|\dot{q}(t)|^{2}\right) d t\right)^{1 / 2} .
$$

Finally, denote by $C_{\text {loc }}^{2}\left(\mathbb{R}, \mathbb{R}^{n}\right)$ the space of $C^{2}$-smooth functions on $\mathbb{R}$ with values in $\mathbb{R}^{n}$ under the topology of almost uniformly convergence of functions and all derivatives up to the order 2 .

TheOREm 1.2. Let $V$ and $f$ satisfy $\left(\mathrm{C}_{1}\right)$ and $\left(\mathrm{C}_{2}\right)$. Assume also that for each $k \in \mathbb{N}$, the Newtonian system (1.2) has a solution $q_{k} \in E_{k}$. If $\left\{\left\|q_{k}\right\|_{E_{k}}\right\}_{k \in \mathbb{N}}$ is a bounded sequence in $\mathbb{R}$ then there exist a subsequence $\left\{q_{k_{j}}\right\}_{j \in \mathbb{N}}$ and a function $q \in E$ such that

$$
q_{k_{j}} \rightarrow q, \quad \text { as } j \rightarrow \infty
$$

in the topology of $C_{\mathrm{loc}}^{2}\left(\mathbb{R}, \mathbb{R}^{n}\right)$ and $q$ is a desired almost homoclinic solution of the Newtonian system (1.1).

In the last twenty years many authors studied homoclinic and heteroclinic solutions of Hamiltonian and Newtonian systems. In particular, homoclinic orbits were considered in [1], [3]-[5], [10], [12], [13]. Many questions are still open (see the survey of P. Rabinowitz [11]). The present paper is partially motivated 
by P. Rabinowitz [9] in which the existence of a nontrivial homoclinic orbit for a Newtonian system

$$
\ddot{q}+V_{q}(t, q)=0
$$

was proved. The functional $V$ considered by Rabinowitz was of the form:

$$
V(t, q)=-\frac{1}{2}(L(t) q, q)+W(t, q)
$$

where $L$ is a continuous $T$-periodic matrix valued function such that $L(t)$ is positive definite and symmetric for every $t \in[0, T]$, and $W \in C^{1}\left(\mathbb{R} \times \mathbb{R}^{n}, \mathbb{R}\right)$ is $T$-periodic in $t$ and satisfies:

$\left(\mathrm{H}_{1}\right) W_{q}(t, q)=o(|q|)$, as $|q| \rightarrow 0$ uniformly with respect to $t$,

$\left(\mathrm{H}_{2}\right)$ there is $\mu>2$ such that for all $t \in \mathbb{R}, q \in \mathbb{R}^{n} \backslash\{0\}$,

$$
0<\mu W(t, q) \leq\left(q, W_{q}(t, q)\right)
$$

Under the above assumptions, a nontrivial homoclinic solution of the Newtonian system (1.3) was obtained as a limit in $C_{\text {loc }}^{2}\left(\mathbb{R}, \mathbb{R}^{n}\right)$ of a certain subsequence of $2 k T$-periodic solutions of this system. The same method was applied in [2].

In [6] we received an essential and interesting generalization of Rabinowitz's result. Namely, we proved the existence of an almost homoclinic solution for (1.1) with $V$ of the form:

$$
V(t, q)=-K(t, q)+W(t, q)
$$

where $W$ and $K$ are $C^{1}$-smooth and $T$-periodic with respect to $t, W$ satisfies $\left(\mathrm{H}_{1}\right)-\left(\mathrm{H}_{2}\right)$ and $K$ satisfies:

$\left(\mathrm{H}_{3}\right)$ the pinching condition, i.e. there are positive constants $b_{1}, b_{2}$ such that for all $(t, q) \in \mathbb{R} \times \mathbb{R}^{n}$,

$$
b_{1}|q|^{2} \leq K(t, q) \leq b_{2}|q|^{2}
$$

$\left(\mathrm{H}_{4}\right)$ for all $(t, q) \in \mathbb{R} \times \mathbb{R}^{n}, K(t, q) \leq\left(q, K_{q}(t, q)\right) \leq 2 K(t, q)$.

Moreover, we assumed that $f$ is bounded, continuous and "sufficiently" small in $L^{2}\left(\mathbb{R}, \mathbb{R}^{n}\right)$ (see $[6]$ for more details).

Writing [7] we noticed that the same scheme as in [6] can be used to prove another existence result for (1.1). Therefore we decided to look more closely at this scheme and to formulate a more general result, i.e. Theorem 1.2.

The paper is divided into three sections. Section 2 provides a detailed proof of Theorem 1.2. In Section 3 an application of this theorem is presented. 


\section{The proof of Theorem $\mathbf{1 . 2}$}

At the begining we recall two basic facts which are necessary to prove Theorem 1.2 (see [6] for their proofs).

FACT 2.1. Let $q: \mathbb{R} \rightarrow \mathbb{R}^{n}$ be a continuous map. If a weak derivative $\dot{q}: \mathbb{R} \rightarrow$ $\mathbb{R}^{n}$ is continuous at a point $t_{0}$ then $q$ is differentiable at $t_{0}$ and

$$
\dot{q}\left(t_{0}\right)=\lim _{t \rightarrow t_{0}} \frac{q(t)-q\left(t_{0}\right)}{t-t_{0}} .
$$

Let $L_{\text {loc }}^{2}\left(\mathbb{R}, \mathbb{R}^{n}\right)$ be a space of functions from $\mathbb{R}$ into $\mathbb{R}^{n}$ locally square integrable.

FACT 2.2. Let $q: \mathbb{R} \rightarrow \mathbb{R}^{n}$ be a continuous map such that $\dot{q} \in L_{\mathrm{loc}}^{2}\left(\mathbb{R}, \mathbb{R}^{n}\right)$. Then for each $t \in \mathbb{R}$ the following inequality holds:

$$
|q(t)| \leq \sqrt{2}\left(\int_{t-1 / 2}^{t+1 / 2}\left(|q(s)|^{2}+|\dot{q}(s)|^{2}\right) d s\right)^{1 / 2} .
$$

Let $L_{2 k T}^{\infty}\left(\mathbb{R}, \mathbb{R}^{n}\right)$ denote a space of $2 k T$-periodic essentially bounded functions from $\mathbb{R}$ into $\mathbb{R}^{n}$ under the standard norm

$$
\|q\|_{L_{2 k T}^{\infty}}:=\operatorname{ess} \sup \{|q(t)|: t \in[-k T, k T]\} .
$$

A direct consequence of the inequality (2.1) is as follows.

FACT 2.3. There is $C>0$ such that for each $k \in \mathbb{N}$ and each $q \in E_{k}$,

$$
\|q\|_{L_{2 k T}^{\infty}} \leq C\|q\|_{E_{k}} .
$$

If $T \geq 1 / 2$ then one can choose $C=\sqrt{2}$.

We divided the proof of Theorem 1.2 into two lemmas.

LEMma 2.4. Let $V$ and $f$ satisfy $\left(\mathrm{C}_{1}\right)$ and $\left(\mathrm{C}_{2}\right)$. Assume also that for each $k \in \mathbb{N}$, the Newtonian system (1.2) has a solution $q_{k} \in E_{k}$. If $\left\{\left\|q_{k}\right\|_{E_{k}}\right\}_{k \in \mathbb{N}}$ is a bounded sequence in $\mathbb{R}$ then there exist a subsequence $\left\{q_{k_{j}}\right\}_{j \in \mathbb{N}}$ and a function $q \in E$ such that

$$
q_{k_{j}} \rightarrow q, \quad \text { as } j \rightarrow \infty
$$

in $C_{\mathrm{loc}}^{1}\left(\mathbb{R}, \mathbb{R}^{n}\right)$.

Proof. At first, let us observe that $\left\{q_{k}\right\}_{k \in \mathbb{N}},\left\{\dot{q}_{k}\right\}_{k \in \mathbb{N}}$ and $\left\{\ddot{q}_{k}\right\}_{k \in \mathbb{N}}$ are uniformly bounded sequences. By assumption, there is $M>0$ such that for every $k \in \mathbb{N}$,

$$
\left\|q_{k}\right\|_{E_{k}} \leq M
$$

Combining (2.2) with (2.3) we get

$$
\left\|q_{k}\right\|_{L_{2 k T}^{\infty}} \leq C M
$$


Since $q_{k}$ is a $2 k T$-periodic solution of $(1.2)$, for every $t \in[-k T, k T)$ we have

$$
\left|\ddot{q}_{k}(t)\right| \leq\left|V_{q}\left(t, q_{k}(t)\right)\right|+\left|f_{k}(t)\right|=\left|V_{q}\left(t, q_{k}(t)\right)\right|+|f(t)| .
$$

From (2.4) and $\left(\mathrm{C}_{1}\right)-\left(\mathrm{C}_{2}\right)$ it follows that there exists $M_{1}>0$ such that for each $k \in \mathbb{N}$

$$
\left\|\ddot{q}_{k}\right\|_{L_{2 k T}^{\infty}} \leq M_{1}
$$

Applying the Mean Value Theorem we have that for every $k \in \mathbb{N}$ and for every $t \in \mathbb{R}$ there is $s_{k} \in[t-1, t]$ such that

$$
\dot{q}_{k}\left(s_{k}\right)=\int_{t-1}^{t} \dot{q}_{k}(s) d s=q_{k}(t)-q_{k}(t-1) .
$$

Hence

$\left|\dot{q}_{k}(t)\right|=\left|\int_{s_{k}}^{t} \ddot{q}_{k}(s) d s+\dot{q}_{k}\left(s_{k}\right)\right| \leq \int_{t-1}^{t}\left|\ddot{q}_{k}(s)\right| d s+\left|q_{k}(t)-q_{k}(t-1)\right| \leq M_{1}+2 C M$, and consequently, for each $k \in \mathbb{N}$,

$$
\left\|\dot{q}_{k}\right\|_{L_{2 k T}^{\infty}} \leq M_{1}+2 C M \equiv M_{2} .
$$

To complete the proof, it is sufficient to notice that $\left\{q_{k}\right\}_{k \in \mathbb{N}}$ and $\left\{\dot{q}_{k}\right\}_{k \in \mathbb{N}}$ are equicontinuous. To this end we show that they satisfy Lipschitz's condition with the same constant independent of $k$. For each $k \in \mathbb{N}$ and for all $t_{1}, t_{2} \in \mathbb{R}$ we get

$$
\left|q_{k}\left(t_{2}\right)-q_{k}\left(t_{1}\right)\right|=\left|\int_{t_{1}}^{t_{2}} \dot{q}_{k}(s) d s\right| \leq M_{2}\left|t_{2}-t_{1}\right|
$$

by (2.6), and analogously

$$
\left|\dot{q}_{k}\left(t_{2}\right)-\dot{q}_{k}\left(t_{1}\right)\right| \leq M_{1}\left|t_{2}-t_{1}\right|
$$

by (2.5). Applying now the Ascolá-Arzeli lemma we receive the claim.

LEMMA 2.5. The function q given by Lemma 2.4 is a desired almost homoclinic solution of the Newtonian system (1.1). Moreover,

$$
q_{k_{j}} \rightarrow q, \quad \text { as } j \rightarrow \infty
$$

in the topology of $C_{\mathrm{loc}}^{2}\left(\mathbb{R}, \mathbb{R}^{n}\right)$.

Proof. At the beginning we show that $q$ satisfies the Newtonian system (1.1). We have

$$
\ddot{q}_{k_{j}}(t)+V_{q}\left(t, q_{k_{j}}(t)\right)=f_{k_{j}}(t)
$$

for every $j \in \mathbb{N}$ and $t \in \mathbb{R}$. Since $q_{k_{j}} \rightarrow q$ and $f_{k_{j}} \rightarrow f$ almost uniformly on $\mathbb{R}$, we get $\ddot{q}_{k_{j}} \rightarrow w$ almost uniformly on $\mathbb{R}$, where $w(t)=f(t)-V_{q}(t, q(t))$. Fix 
$a, b \in \mathbb{R}$ and assume that $a<b$. There is $j_{0} \in \mathbb{N}$ such that for every $j>j_{0}$, $[a, b] \subset\left[-k_{j} T, k_{j} T\right)$. Hence for every $j>j_{0}$ and $t \in[a, b]$ we have

$$
\ddot{q}_{k_{j}}(t)+V_{q}\left(t, q_{k_{j}}(t)\right)=f(t) .
$$

By this we get that $\ddot{q}_{k_{j}}$ is continuous in $[a, b]$ for $j>j_{0}$. From Fact 2.1 we conlude that $\ddot{q}_{k_{j}}$ is a derivative of $\dot{q}_{k_{j}}$ in $(a, b)$ for every $j>j_{0}$. Since $\dot{q}_{k_{j}} \rightarrow \dot{q}$ and $\ddot{q}_{k_{j}} \rightarrow w$ almost uniformly on $\mathbb{R}$, we obtain $\ddot{q}=w$ in $(a, b)$. In consequence, $\ddot{q}=w$ in $\mathbb{R}$ and $q$ is a solution of (1.1). Moreover, $\left\{q_{k_{j}}\right\}_{j \in \mathbb{N}}$ goes to $q$ in the topology of $C_{\text {loc }}^{2}\left(\mathbb{R}, \mathbb{R}^{n}\right)$.

Now we notice that $q$ emanates from 0 and terminates at 0 . We have

$$
\begin{aligned}
\int_{-\infty}^{\infty}\left(|q(t)|^{2}+|\dot{q}(t)|^{2}\right) d t & =\lim _{m \rightarrow \infty} \int_{-m T}^{m T}\left(|q(t)|^{2}+|\dot{q}(t)|^{2}\right) d t \\
& =\lim _{m \rightarrow \infty} \lim _{j \rightarrow \infty} \int_{-m T}^{m T}\left(\left|q_{k_{j}}(t)\right|^{2}+\left|\dot{q}_{k_{j}}(t)\right|^{2}\right) d t
\end{aligned}
$$

By (2.3), for each $m \in \mathbb{N}$ there is $j(m) \in \mathbb{N}$ such that for all $j>j(m)$,

$$
\int_{-m T}^{m T}\left(\left|q_{k_{j}}(t)\right|^{2}+\left|\dot{q}_{k_{j}}(t)\right|^{2}\right) d t \leq M^{2} .
$$

Hence

$$
\int_{-\infty}^{\infty}\left(|q(t)|^{2}+|\dot{q}(t)|^{2}\right) d t \leq M^{2}
$$

and, in consequence,

$$
\int_{|t| \geq r}\left(|q(t)|^{2}+|\dot{q}(t)|^{2}\right) d t \rightarrow 0,
$$

as $r \rightarrow \infty$. Combining (2.7) with (2.1), we get $q(t) \rightarrow 0$, as $t \rightarrow \pm \infty$, which completes the proof.

\section{Application}

Let us consider the Newtonian system

$$
\ddot{q}-V_{q}(t, q)=f(t),
$$

where $t \in \mathbb{R}, q \in \mathbb{R}^{n}$. We will assume that $V: \mathbb{R} \times \mathbb{R}^{n} \rightarrow \mathbb{R}$ and $f: \mathbb{R} \rightarrow \mathbb{R}^{n}$ satisfy $\left(\mathrm{C}_{1}\right)-\left(\mathrm{C}_{2}\right)$, and moreover,

$\left(\mathrm{C}_{3}\right) V(t, q) \geq b(t)|q|^{2}$ for all $(t, q) \in \mathbb{R} \times \mathbb{R}^{n}$, where $b: \mathbb{R} \rightarrow \mathbb{R}_{+}$is a continuous positive-valued function that achieves a minimum,

$\left(\mathrm{C}_{4}\right) V(t, 0)=0$ for every $t \in \mathbb{R}$.

Notice that if $b \in C_{T}\left(\mathbb{R}, \mathbb{R}_{+}\right)$then $b$ has a minimum. 
THEOREM 3.1. Under the assumptions $\left(\mathrm{C}_{1}\right)-\left(\mathrm{C}_{4}\right)$, the Newtonian system (3.1) possesses an almost homoclinic solution.

Our aim is to prove Theorem 3.1 by using Theorem 1.2. An approximative sequence of second order differential equations for the Newtonian system (3.1) is as follows:

$$
\ddot{q}-V_{q}(t, q)=f_{k}(t),
$$

where $t \in \mathbb{R}, q \in \mathbb{R}^{n}$ and for each $k \in \mathbb{N}, f_{k}: \mathbb{R} \rightarrow \mathbb{R}^{n}$ is a $2 k T$-periodic extension of $f_{\mid[-k T, k T)}$ onto $\mathbb{R}$.

For each $k \in \mathbb{N}$, let $I_{k}: E_{k} \rightarrow \mathbb{R}$ be given by

$$
I_{k}(q):=\int_{-k T}^{k T}\left(\frac{1}{2}|\dot{q}(t)|^{2}+V(t, q(t))+\left(f_{k}(t), q(t)\right)\right) d t .
$$

It is well-known that for a fixed $k \in \mathbb{N}$ critical points of the functional $I_{k}$ are classical $2 k T$-periodic solutions of (3.2). In order to show that all assumptions of Theorem 1.2 are fulfilled, we need a standard minimizing argument, i.e. the following theorem.

Theorem 3.2 (see $[8$, Theorem 1.1]). If $\varphi: X \rightarrow \mathbb{R}$ is a weakly lower semicontinuous functional on a reflexive Banach space $X$ and has a bounded minimizing sequence, then $\varphi$ has a minimum on $X$.

The existence of a bounded minimizing sequence will be in particular insured when $\varphi$ is coercive, i.e. such that

$$
\varphi(x) \rightarrow \infty, \quad \text { if }\|x\| \rightarrow \infty .
$$

Proof of Theorem 3.1. Let us define $B:=\min _{t \in \mathbb{R}} b(t), A:=\min \{1 / 2, B\}$ and $L:=\|f\|_{L^{2}\left(\mathbb{R}, \mathbb{R}^{n}\right)}$. It is obvious that for each $k \in \mathbb{N}$,

$$
\left\|f_{k}\right\|_{L_{2 k T}^{2}} \leq L
$$

Applying $\left(\mathrm{C}_{3}\right)$ we receive

$$
\begin{aligned}
I_{k}(q) & \geq \int_{-k T}^{k T}\left(\frac{1}{2}|\dot{q}(t)|^{2}+b(t)|q(t)|^{2}+\left(f_{k}(t), q(t)\right)\right) d t \\
& \geq A\|q\|_{E_{k}}^{2}+\int_{-k T}^{k T}\left(f_{k}(t), q(t)\right) d t \\
& \geq A\|q\|_{E_{k}}^{2}-\left\|f_{k}\right\|_{L_{2 k T}^{2}}\|q\|_{E_{k}} \geq A\|q\|_{E_{k}}^{2}-L\|q\|_{E_{k}} .
\end{aligned}
$$

Hence $I_{k}$ is a functional bounded from below and coercive. 
Assume that $q_{m} \rightarrow q$ in $E_{k}$. Then $\dot{q}_{m} \rightarrow \dot{q}$ in $L_{2 k T}^{2}\left(\mathbb{R}, \mathbb{R}^{n}\right)$. Since the square of norm in a Hilbert space is weakly lower semicontinuous, we conclude that the functional given by

$$
E_{k} \ni q \mapsto \int_{-k T}^{k T} \frac{1}{2}|\dot{q}(t)|^{2} d t=\frac{1}{2}\|\dot{q}\|_{L_{2 k T}^{2}}^{2}
$$

is weakly lower semicontinuous, too. Moreover, $q_{m} \rightarrow q$ almost uniformly on $\mathbb{R}$. Therefore

$$
\int_{-k T}^{k T}\left[V\left(t, q_{m}(t)\right)+\left(f_{k}(t), q_{m}(t)\right)\right] d t \rightarrow \int_{-k T}^{k T}\left[V(t, q(t))+\left(f_{k}(t), q(t)\right)\right] d t,
$$

as $m \rightarrow \infty$, and so the functional defined by

$$
E_{k} \ni q \mapsto \int_{-k T}^{k T}\left[V(t, q(t))+\left(f_{k}(t), q(t)\right)\right] d t
$$

is weakly continuous. In consequence, we get that $I_{k}$ is weakly lower semicontinuous. Finally, from Theorem 3.2 it follows that $I_{k}$ achieves a minimum on $E_{k}$. For every $k \in \mathbb{N}$ there is $q_{k} \in E_{k}$ such that

$$
I_{k}\left(q_{k}\right)=\min _{q \in E_{k}} I_{k}(q) \quad \text { and } \quad I_{k}^{\prime}\left(q_{k}\right)=0 .
$$

Set

$$
\varrho:=\frac{L+\sqrt{L^{2}+4 A}}{2 A} .
$$

Clearly, $\varrho$ is independent of $k$. By $\left(\mathrm{C}_{4}\right)$, for each $k \in \mathbb{N}$, we have $I_{k}(0)=0$. From (3.3) we get that for each $k \in \mathbb{N}$, if $\|q\|_{E_{k}} \geq \varrho$ then $I_{k}(q) \geq 1$. Thus

$$
\left\|q_{k}\right\|_{E_{k}}<\varrho
$$

for every $k \in \mathbb{N}$. Applying now Theorem 1.2 we receive our claim.

\section{REFERENCES}

[1] A. Ambrosetti and V. Coti Zelati, Multiple homoclinic orbits for a class of conservative systems, Rend. Sem. Mat. Univ. Padova 89 (1993), 177-194.

[2] F. Antonacci And P. Magrone, Second order nonautonomous systems with symmetric potential changing sign, Rend. Mat. Appl. (7) 18 (1998), 367-379.

[3] T. BARTSCH AND A. SzUlkin, Hamiltonian systems: periodic and homoclinic solutions by variational methods, Handbook of Differential Equations, vol. 2, Elsevier B. V., Amsterdam, 2005, pp. 77-146.

[4] Y. Ding And M. Girard, Periodic and homoclinic solutions to a class of Hamiltonian systems with the potentials changing sign, Dynam. Systems Appl. 2 (1993), 131-145.

[5] Y. Ding And S. J. Li, Homoclinic orbits for first order Hamiltonian systems, J. Math. Anal. Appl. 189 (1995), 585-601.

[6] M. IZYDOREK AND J. JANCZEWSKA, Homoclinic solutions for a class of the second order Hamiltonian systems, J. Differential Equations 219 (2005), 375-389. 
[7]_ Homoclinic solutions for nonautonomous second order Hamiltonian systems with a coercive potential, J. Math. Anal. Appl. 335 (2007), 1119-1127.

[8] J. Mawhin and M. Willem, Critical point theory and Hamiltonian systems, Applied Mathematical Sciences, vol. 74, Springer-Verlag, New York, 1989.

[9] P. H. Rabinowitz, Homoclinic orbits for a class of Hamiltonian systems, Proc. Roy. Soc. Edinburgh Sect. A 114 (1990), 33-38.

[10] Connecting orbits for a reversible Hamiltonian system, Ergodic Theory Dynam. Systems 20 (2000), 1767-1784.

[11] Variational methods for Hamiltonian systems, Handbook of Dynamical Systems, vol. 1A, Elsevier, Amsterdam, 2002, pp. 1091-1127.

[12] E. SÉRÉ, Existence of infinitely many homoclinic orbits in Hamiltonian systems, Math. Z. 209 (1992), 27-42.

[13] A. Szulkin And W. Zou, Homoclinic orbits for asymptotically linear Hamiltonian systems, J. Funct. Anal. 187 (2001), 25-41.

Manuscript received December 31, 2007

JOANNA JANCZEWSKA

Faculty of Technical Physics and Applied Mathematics

Gdańsk University of Technology

G. Narutowicza 11/12

80-952 Gdańsk, POLAND

and

Institute of Mathematics

Polish Academy of Sciences

Śniadeckich 8

00-956 Warszawa, POLAND

E-mail address: janczewska@mifgate.pg.gda.pl,j.janczewska@impan.pl 